\title{
Packet Transfer Analysis between Vehicles using AODV in VANET
}

\author{
Deepak Gupta \\ Research Scholar \\ Deptt of Computer \\ Science
}

Govt. Engg College, Ajmer

\author{
Rakesh Rathi, PhD \\ Assistant Professor \\ Deptt of Computer \\ Science
}

Govt. Engg College, Ajmer

\author{
Shikha Gupta \\ Research Scholar \\ Deptt of Computer \\ Science
}

\author{
Neetu Sharma, PhD \\ Assistant Professor \\ Deptt of Computer \\ Science
}

Govt. Engg College, Ajmer Govt. Engg College, Ajmer

\begin{abstract}
Vehicular Ad-Hoc Network or VANET is a subset of Mobile Ad-Hoc Network or MANET that provides communication between vehicles and between vehicles and road-side units with an aim of providing efficient and safe transportation. A vehicle in VANET is considered to be an intelligent mobile node which is able to communicate with its neighbours and other vehicles in the network. VANET introduces more challenges as compare to MANET because of high mobility (Movement) of nodes and fast topology changes in VANET. It ensures that established routing paths do not break before the end of data transmission. Various routing protocols have been designed and presented by researchers after considering the major challenges involved in VANET. This paper provides a survey of Ad hoc On-Demand Distance Vector or AODV routing protocol in urban city traffic scenario using SUMO and network performance using NS2 using network parameters such as packet delivery ratio, throughput and delay for VANET.
\end{abstract}

\section{Keywords}

VANET, AODV, SUMO, NS2, MOVE, XGraph, Mobility Simulator

\section{INTRODUCTION}

VANET follows a basic concept which consists of a large web of vehicles and a wireless local area network (WLAN) technology which connects all the vehicles together to make communication possible. The main aim of VANETs is to build an Intelligent Transport System (ITS). The safetyrelated applications are the main aim of inter-vehicular communication. When an accident is detected, a vehicle can continuously broadcast information about this tough situation to the other vehicles or when a vehicle brakes suddenly, it gives information about the other vehicles to the driver.

Although Vehicular Ad-hoc Network (VANET) is not a new topic. The main objective of VANET is to help a group of vehicles to set up and maintain a communication network among them without using any central base station or any controller. However, along with these useful applications of VANET, creates new challenges and problems. Lack of infrastructure in VANET puts additional responsibilities on vehicles. Every vehicle becomes part of the network and also manages and controls the communication on this network along with its own communication requirements. Vehicular ad-hoc networks are responsible for the communication between moving vehicles in a certain environment. A vehicle can communicate with another vehicle directly which is called Vehicle to Vehicle (V2V) communication, or a vehicle can communicate to an infrastructure such as a Road Side Unit (RSU), known as Vehicle-to-Infrastructure (V2I).

\section{CHARACTERSTICS}

Following are some characteristics of VANET: -

i. High Mobility: The nodes present in VANETs move at a very high speed.

ii. High Dynamic Topology: The topology of VANET changes dynamically because of the movement of vehicles at high speed.

iii. Battery Power and Storage Capacity: Power constraint always exists in various networks but in VANETs vehicles are able to provide power to on board unit (OBU) via the long life battery.

iv. Unbounded Network Size: The network size in VANET is geographically unbounded because it can be generated for one city or one country.

v. Time Critical: Timely delivery of information is very essential. Actions when it is required.

vi. Frequent changing information: Ad-Hoc nature of VANET motivates the nodes to gather information from other vehicles and roadside units. As vehicles move and change their path information related to traffic and environment also changes very rapidly.

vii. Wireless Communication: Moving vehicles or nodes are connected and exchange their information through wireless.

\section{ROUTING PROTOCOL-AODV}

A routing protocol use software and routing algorithms to find best network to data transfer and communication paths between nodes. Ad hoc on-demand Distance Vector (AODV) is one of the commonly used reactive on demand routing protocols in ad hoc network. AODV is a reactive extension of the DSDV protocol. In AODV, along with routing tables of every node, two counters including Sequence Number (SEQ NO) and broadcast ID are maintained also. The destination IP is already known to which data is to be transferred from source. Thus, the destination Sequence Number (SEQ NO) helps to determine an updated path from source to destination. Route Request (RREQ) and Route Response (RRESP) packets are used in which RREQ is responsible for finding routes from source to destination and RRESP sends back the route information response to its source. In Ad-Hoc On Demand Distance Vector Routing, the source node and destination nodes IP addresses are already known. The goal is to identify, find and maintain the optimal route between source and destination node in order to send/receive data packets and informative [10]. Each node comprises of a routing table along with the following fields of Route Request (RREQ) packet. 
RREQ \{ Destination IP, Destination Sequence Number, Source IP, Source Sequence Number, Hop Count\}

Below figure shows the basic working of AODV Network

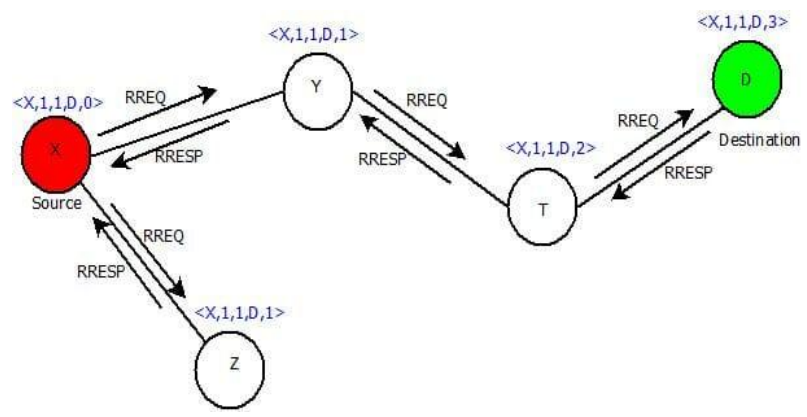

Fig 1: AODV Network[12]

\section{ENVIRONMENT SETUP}

Here we are using SUMO, MOVE and NS 2.35 for packet transfer analysis in moving vehicles using AODV in VANET.
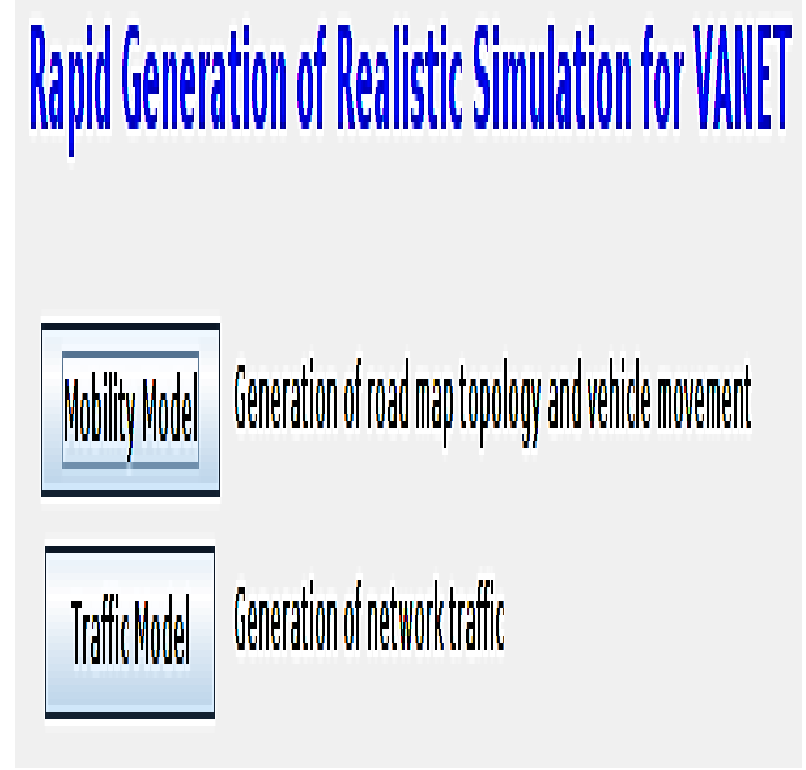

Fig 2: Simulation Methods[9]

The simulation of VANETs requires two different components: Mobility model generator and Traffic model generator.

i. Mobility Model Generator: Mobility model simulation was generated by using MOVE (Mobility model generator for Vehicular networks) with SUMO (Simulator for Urban Mobility) as a VANETs simulator.

\section{Mobility Model Generator for VANET}

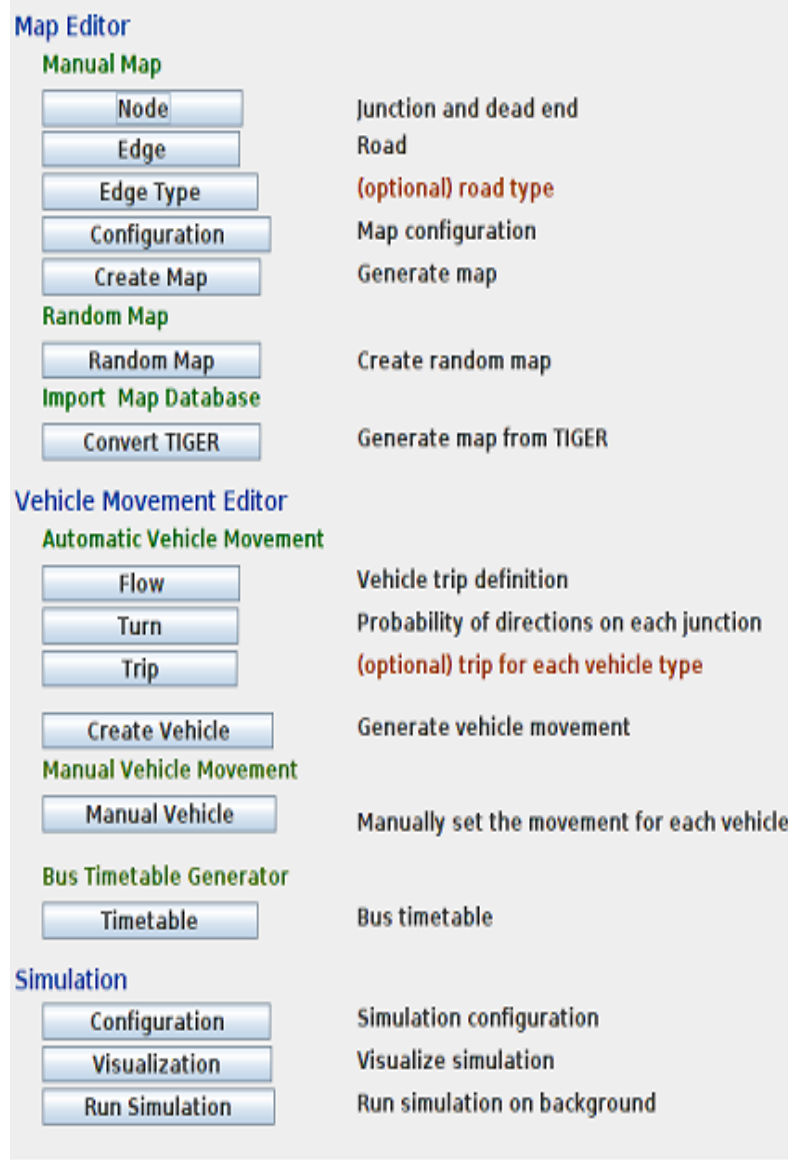

Fig 3: Mobility Model Generator for VANET[9]

SUMO: SUMO is an open source traffic simulation package which was initiated in year 2001 [10]. We can use SUMO to build a Network and assign routes within a network, Develop traffic light algorithm, Simulate and analyse traffic within a network, given Origin-Destination (OD) Matrix. In SUMO, road network can be created in many ways:

1. Manually by creating our own node, edge, routeconnection files.

2. Using netgenerate command.

3. Importing road network from external sources such as OSM.

For our packet analysis in VANET, we are manually creating the nodes and edges. Our Route Assignment is as follows:

Node file creation (.nod.xml), Edge file creation (.edg.xml), Edge type file creation (.type.xml), Network creation from the node, edge and type files, Route file (.rou.xml).

NS2: Network Simulation (NS) is one of the types of simulation, which is used to simulate the network such as in MANETs, VANETs etc. It provides simulation for routing and multicast protocols for both wired and wireless networks. NS is licensed for use under version 2 of the GNU (General Public License) and is popularly known as NS2. It is objectoriented, discrete event-driven simulator written in $\mathrm{C}++$ and OTCL/TCL [1].

The methodology steps that were used to generate mobility and traffic model are shown below: 


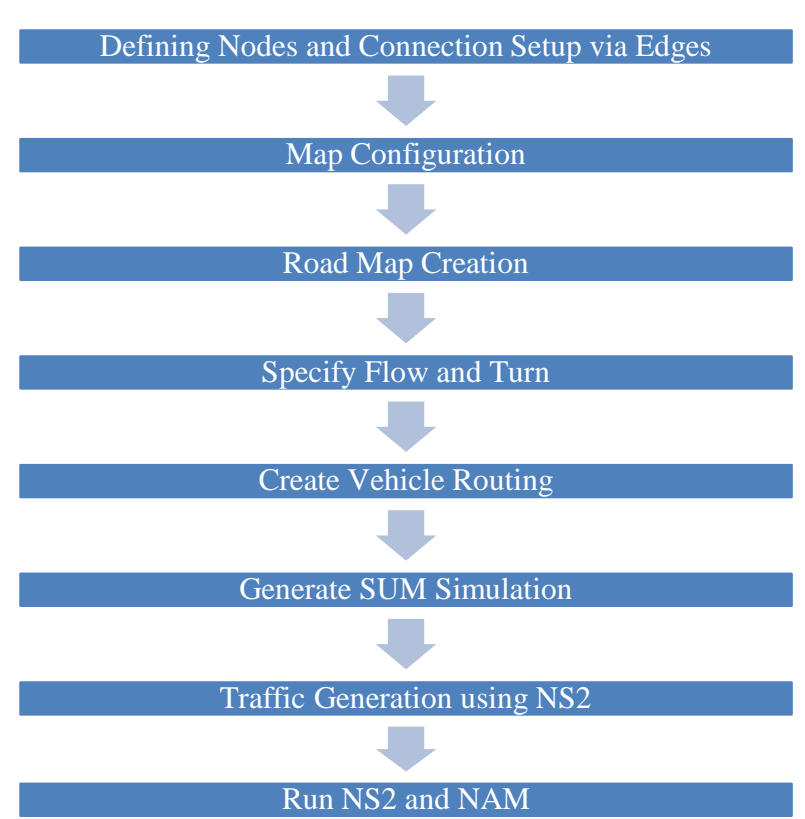

Fig 4: Applied Methodology

After entering all the past information as an input in the Map Editor and Vehicle Movement Editor using following command:

\section{Java -jar MOVE.jar}

This information is fed into sumo in order to generate traffic mobility trace as shown below:

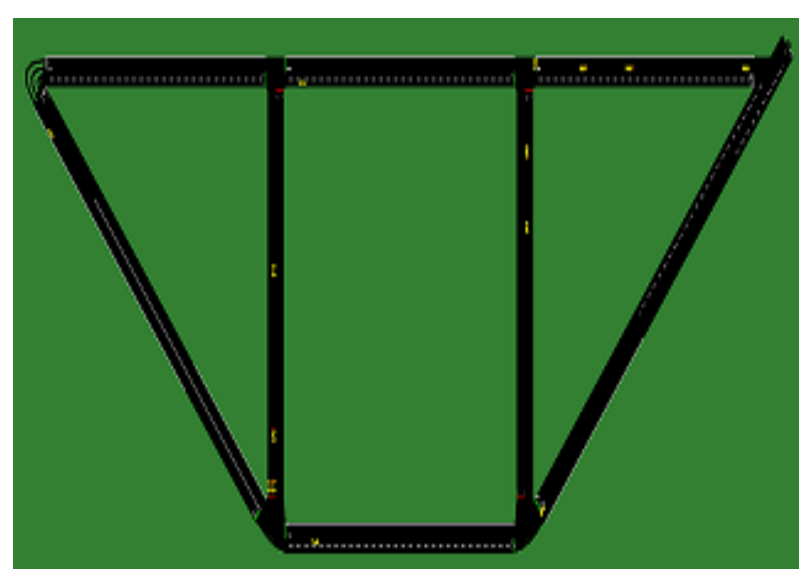

Fig 5: Traffic Mobility Trace File in SUMO[9]Output of simulation process is shown below:

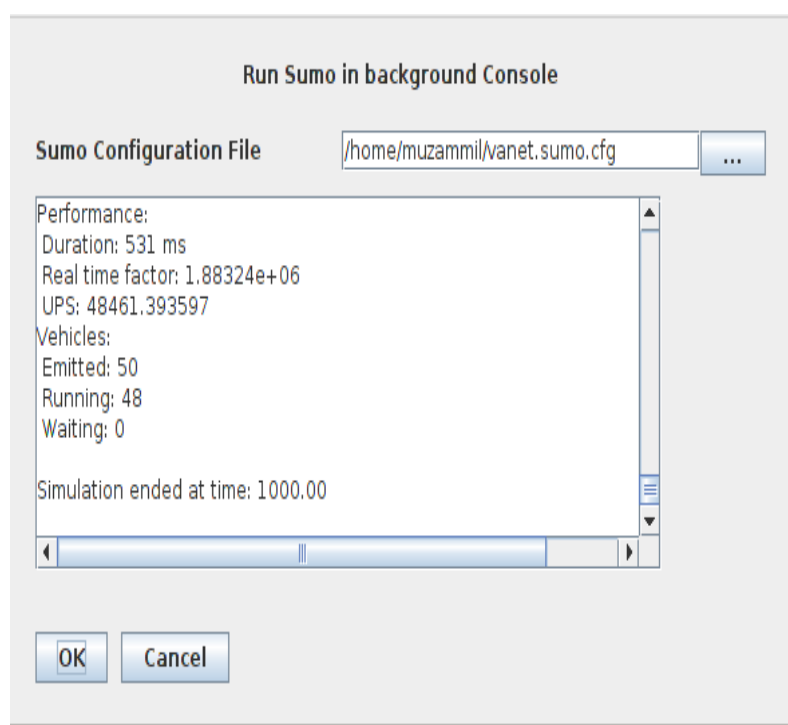

Fig 6: Output of SUMO Configuration file

ii. Traffic Model Generator: To analyse the vehicular ad hoc network characteristics and protocol performances, traffic simulators are require to generate position and movement information of each and every vehicle in VANETs environment. MOVE tool has traffic mobility generator that can work with both NS2 and Qualnet as shown in below figure.

\section{Trafific Model Generatoor for VANET}

NS.2

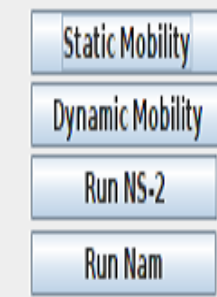

Qualnet

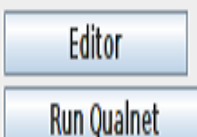

Static Traffic Model Generator for NS.2 (w/o TraCl) Dynamic Traffic Model Generator for NS.2 (w TraCl) Run NS.2in console Visualize NS.2 Traffic Model Generator for Qualnet Run Qualnet in console
Fig 7: Traffic Model in MOVE

In this work, NS2 simulation is used to deal with static traffic simulation file and generate tcl file as shown below: 


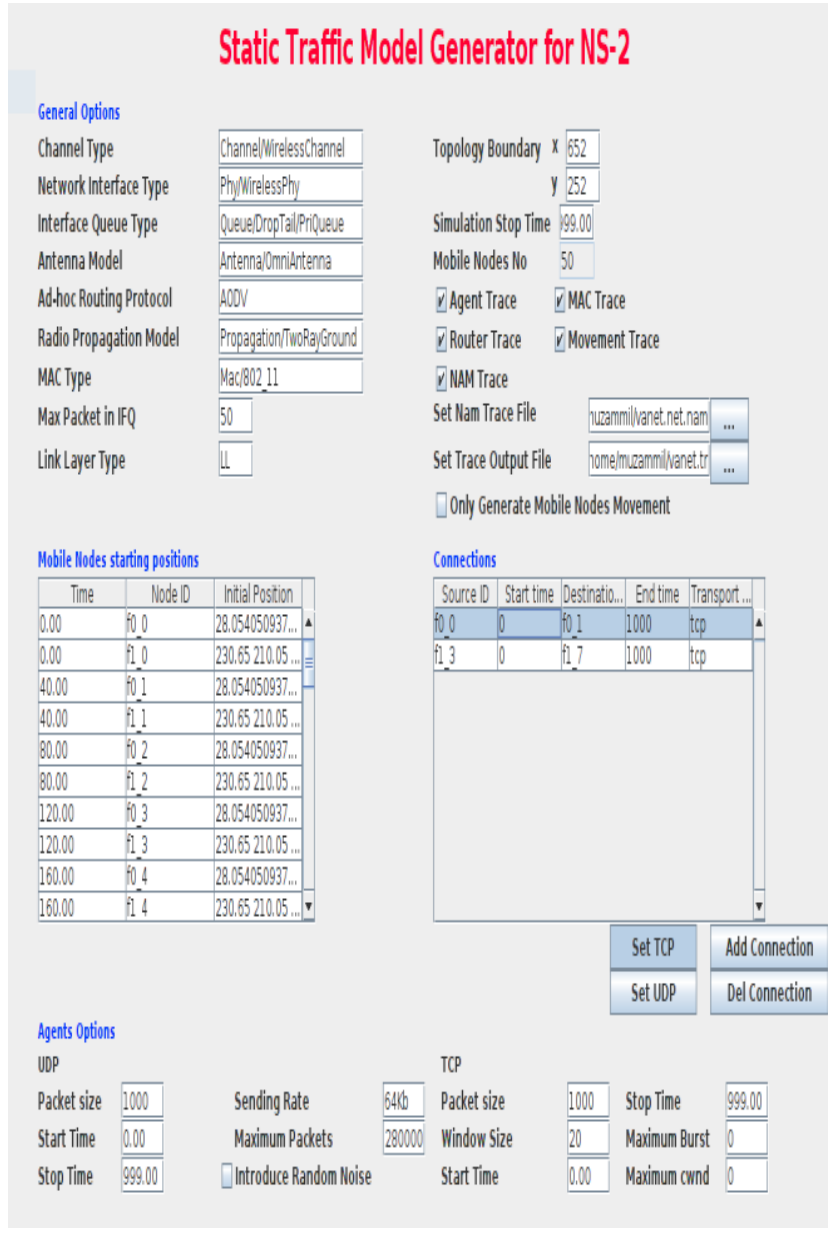

Fig 8: Static Traffic Model Generator for Road Map

The tcl file is generated from both MOVE trace file and road map. The output of this tcl file is shown below:

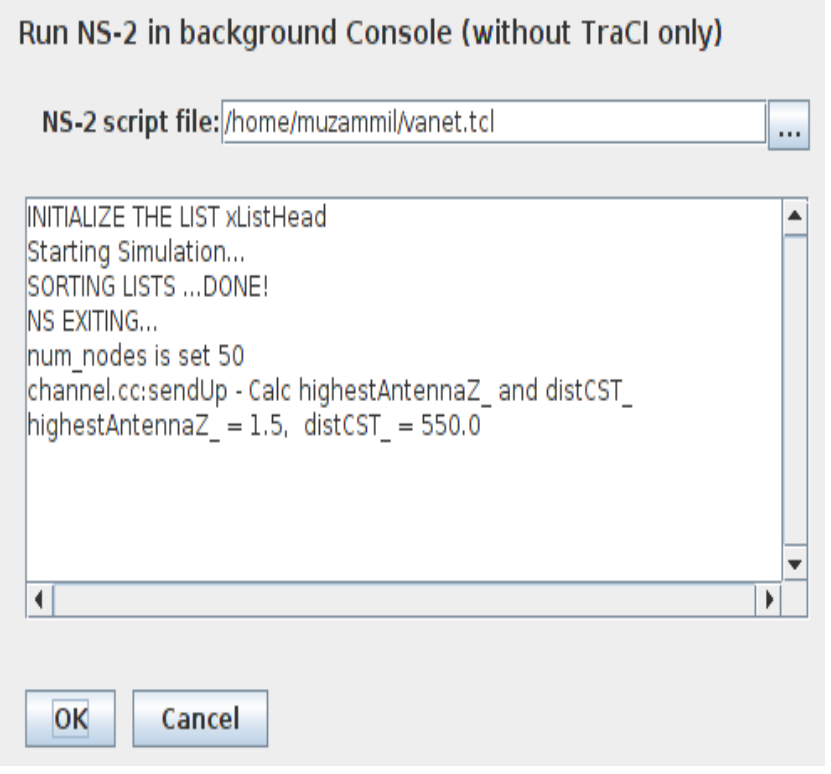

Fig 9: Output of TCL file

Finally NAM file is generated and the output is shown below:

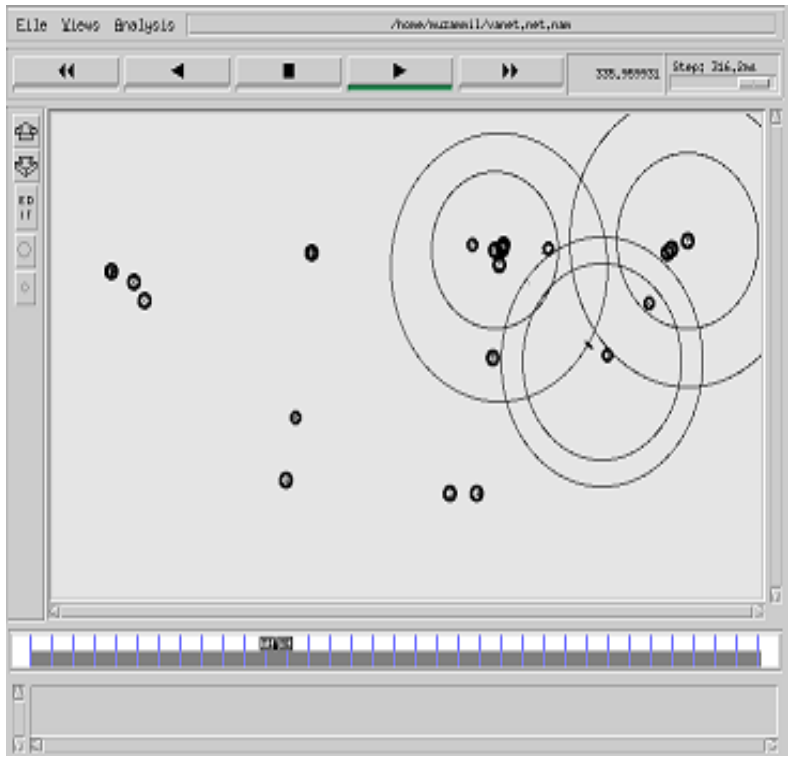

Fig 10: NAM file for proposed Mobility

\section{SIMULATION RESULTS}

For all the simulations, the same movement models were used, the number of traffic sources was fixed at 20, the maximum speed of the nodes was set to $20 \mathrm{~m} / \mathrm{s}$ and the pause time was varied as $0 \mathrm{~s}, 10 \mathrm{~s}, 20 \mathrm{~s}, 40 \mathrm{~s}$ and $100 \mathrm{~s}$.

Packet Delivery Fraction: The ratio of the data packets delivered to the destinations to those generated by the Continuous Bit Rate (CBR) sources is known as packet delivery fraction. The On-demand protocol AODV performed particularly well, delivering over $85 \%$ of the data packets regardless of mobility rate. Packet Delivery Fraction is calculated as total packets received by all nodes in the destination to total packets send by all source nodes. The following Xgraph shows the effect of increasing the number of vehicles for proposed mobility in VANET.

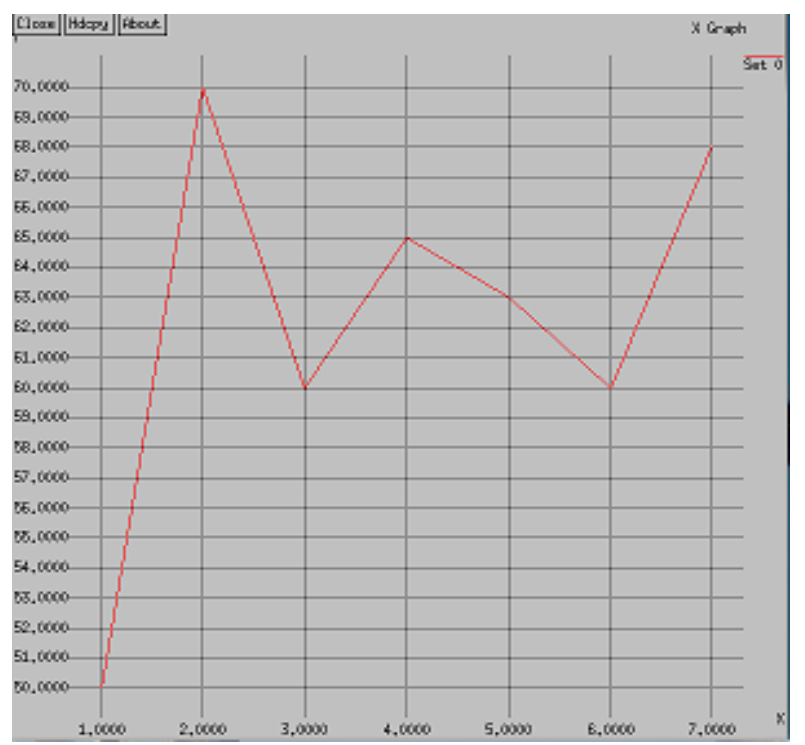

Fig 11: Number of Vehicles Vs Packet Delivery Fraction

Average End To End Delay: Average end to end delay includes all possible delays caused by buffering during route discovery latency, queuing at the interface queue, retransmission delays at the MAC, and propagation and transfer times of data packets. The average end-to-end delay of 
packet delivery was lower in AODV as compared to other protocols. All of the protocols deliver a greater percentage of the originated data packets when there is little node mobility (i.e., at large pause time), converging to $100 \%$ delivery when there is no node motion. An attempt was made to evaluate the performance difference between the two by varying the Mobility pattern and Number of traffic sources. The Delay is calculated as:

Delay $=$ Number of sending bits in the packet/Throughput Where throughput is defined as the packets per unit time interval length, and Delay represents the time period that needs to route a packet from the source to the desired destination.

\section{PERFORMANCE ANALYSIS AND EFFECT OF MOBILITY}

The simulation results bring out some important characteristic differences between the routing protocols. The presence of high mobility creates frequent link failures and each routing protocol reacts differently during link failures. The different basic working mechanism of these protocols leads to the differences in the performance. For AODV, packet delivery ratio is independent of offered traffic load, delivering between $85 \%$ and $100 \%$ of the packets in all cases. In contrast, the approach used by the on-demand protocol, AODV to build the routing information as and when they are created make them more adaptive and result in better performance (high packet delivery fraction and lower average end-to-end packet delays). In the presence of high mobility, link failures can happen very frequently. Link failures trigger new route discoveries in AODV since it has at most one route per destination in its routing table. Thus, the frequency of route discoveries in AODV is directly proportional to the number of route breaks. In lower mobility scenarios, other protocols often perform better than AODV, because the chances of find the route in one of the caches is much higher. However, due to the constrained simulation environment (lesser simulation time and lesser mobility models), the better performance of AODV couldn't be observed.

\section{CONCLUSIONS}

This paper analyses the performance of AODV routing protocol for ad hoc networks using ns2 simulations. In most of the cases AODV perform better under high mobility simulations. High mobility results in frequent link failures and the overhead involved in updating all the nodes with the new routing information is much more than that involved in AODV, where the routes are created as and when required. AODV uses routing tables, one route per destination, and destination sequence numbers, a mechanism to prevent loops and to determine freshness of routes. The general observation from the simulation is that for application-oriented metrics such as packet delivery fraction and delay AODV, outperforms in more "stressful" situations (i.e., smaller number of nodes and lower load and/or mobility), with widening performance gaps with increasing stress (e.g., more load higher mobility).

\section{REFERENCES}

[1] Simulation tools download from https://www.nsnam.org.

[2] Samyak Shah, Amit Khandre, Mahesh Shirole and Girish Bhole, Performance Evaluation of Ad Hoc Routing Protocols Using NS2 Simulation.

[3] NS-2, The ns Manual (formally known as NS Documentation) available at http: //www isi.edu/nsnam/ns/doc.

[4] Ian D. Chakeres and Elizabeth M. Belding-Royer. AODV Routing Protocol Implementation Design

[5] Gao Fang Lu, Yuan Zhang, Qing shun Li Chunli,"Simulation and Analysis for the Performance of the Mobile Ad Hoc Network Routing Protocols,"The Eighth International Conference on Electronic Measurement and Instruments, 2007.

[6] Surinder Singh et al, International Journal of Computer Science and Mobile Computing, Vol.5 Issue.6, June2016, pg. 434-441

[7] F. D. da Cunha, A. Boukerche, L. Villas, V. Aline, and A. A. F. Loureiro, "Data communication in VANETs: a survey, challenges and applications," Research Report RR-8498, Version 2, INRIA, 2014

[8] International Journal of Computer Applications (0975 8887)Volume 123 -No.10, August 2015 Vehicular Ad hoc Network sand its Applications in Diversified Fields Raju Barskar, Meenu Chawla

[9] https://www.nsnam.com/2014/09/sumo-and-moveexample-traffic-simulation.html

[10] SUMO version from https://sumo.dlr.de/wiki/Downloads

[11] Aijaz Ahmad Anchari et al, "Routing Problems in Mobile Ad hoc Networks (MANET)" International Journal of Computer Science and Mobile Computing, Vol.6 Issue.7, July-2017, pg. 9-15

[12] https://www.minigranth.com/ mobile- adhoc- networks/ aodv/ 